CP, 2018, Vol.7 - No15, pp. 137/153 ISSN 2014-6752. Girona (Catalunya). Universitat de Girona. CASTILLO-ESPARCIA, Antonio y VILLABONABELTRÁN, Diego: Evaluación para demostrar la efectividad en comunicación y relaciones públicas. Una mirada a las prácticas profesionales desde los Communication Monitor. Recibido: 21/05/2018 - Aceptado: 15/10/2018

\title{
Evaluación para demostrar la efectividad en comunicación y relaciones públicas. Una mirada a las prácticas profesionales desde los Communication Monitor
}

\section{Evaluation to demonstrate effectiveness in communication and public relations. A look at professional practices from the Communication Monitor}

\author{
AUTORES: \\ Dr. Antonio Castillo-Esparcia \\ Universidad de Málaga, acastilloe@uma.es \\ http://orcid.org/0000-0002-9751-8628 \\ Diego Villabona-Beltrán \\ Universidad de Málaga, dvillabona@gmail.com \\ https://orcid.org/0000-0002-7409-1828
}

\section{Resumen}

Las organizaciones invierten grandes cantidades de recursos económicos y humanos para escuchar y entender a sus públicos buscando establecer relaciones dialógicas, de confianza y credibilidad en medio de un competitivo entorno empresarial. Frente a ello, los profesionales de la comunicación están siendo presionados para generar resultados sobre los públicos que puedan ser medibles a partir de variables como conocimiento, recordación y recomendación de los mensajes, motivación a la acción y cambio de comportamientos o percepciones en función de los intereses corporativos.

Frente a esta exigencia es la evaluación la que permitirá a los profesionales de la comunicación demostrar el valor y la efectividad que generan sus actividades. A partir de una revisión de los resultados publicados en los Communication Monitor en Europa, Asia-Pacífico y América Latina por medio del análisis de contenido como técnica de interpretación

\section{Abstract}

Organizations invest large amounts of economic and human resources to listen and understand their audiences by establishing credible, trustworthy and conversational relationships in a competitive business environment. With regard to this, communication and public relations professionals are being pressured to generate results about the public that can be measured based on variables such as knowledge, consciousness and recommendation of messages, motivation to action, and modification of behaviors or perceptions related to corporate interests.

Confronted with this requirement, evaluation will allow communication and public relations professionals to demonstrate the value and effectiveness generated by their activities. Found on a review of the results published in Communication Monitor in Europe, Asia-Pacific and Latin America, an approach is made for trends and recent considerations in professional practices in communication 
de textos e instrumento de recogida de información; se realiza un acercamiento a las tendencias y consideraciones recientes en las prácticas profesionales en evaluación de comunicación, el nivel de implementación de esta fase en las estrategias y programas de comunicación, el estado de avance que ha tenido, los niveles de medición más utilizados, las acciones y medios que son objeto de mayor medición por parte de los profesionales y las principales exigencias en términos de resultados que las organizaciones esperan del ejercicio de la comunicación y el impacto que tiene en los diferentes públicos.

Palabras claves: evaluación; medición; comunicación estratégica; relaciones públicas. evaluation, the level of implementation of this phase in the strategies and communication programs, the state of progress it has had, the most used levels of measurement, the actions and means that are subject to greater measurement by professionals, and the main demands in terms of results that organizations expect from the exercise of communication and the impact it holds on different publics.

Keywords: evaluation; measurement; strategic communication; public relations.

\section{Introducción}

Bajo un enfoque de planificación estratégica, fundamentado en las etapas del conocido modeIo RACE (research, action, communication, evaluation) formulado para la toma de decisiones en comunicación, nos centraremos específicamente en la última fase, la de evaluación, debido a que ha sido la menos desarrollada y en la actualidad muy pocas organizaciones evalúan su desempeño en comunicación en relación a los demás procesos de gestión y fundamentalmente, en función de los objetivos organizacionales según lo plantean las investigaciones de Castillo y Nobell (2015) que al citar a Xifra (2005) sostienen también que la investigación y su posterior evaluación constituyen las etapas más esenciales de todo el proceso de planificación estratégica que se vuelve fundamental más aún cuando la comunicación adquiere una función directiva en las organizaciones.

Al respecto Zerfass, Verčič y Volk (2017a citando a Watson, 2012, p. 394; Yin et al., 2012, p. 42) plantearán que las organizaciones invierten grandes cantidades de recursos financieros y humanos para alcanzar y escuchar a sus públicos en medio de un competitivo entorno empresarial. Con los presupuestos en aumento y un número creciente de canales, los profesionales de la comunicación están siendo presionados gradualmente para proporcionar "hechos concretos" en lugar de decisiones de invertir en comunicación basadas en la intuición o la experiencia.

Frente a esta exigencia es la evaluación la que permite a los profesionales de la comunicación demostrar el valor de sus actividades para sus organizaciones (Zerfass et al., 2017a citando a Stacks y Michaelson, 2014; Watson y Noble, 2014) y responder a la alta dirección que está exigiendo conocer los resultados y el retorno a la inversión de sus asignaciones presupuestarias (Gregory, 2001 citado por Marca, Oliveira, Matilla y Miranda, 2017). 
Señala también Marca et al., (2017) que la evaluación de los programas de comunicación desempeña un papel importante en la rendición de cuentas y en la demostración de la eficacia, así como en el impacto en la estrategia corporativa y de negocio de toda organización. Al citar a Gregory (1996) exponen que, si se consigue el logro de los objetivos, nadie puede dudar del valor del profesional, ya que el adecuado uso de la evaluación demuestra la efectividad y la eficacia de la inversión realizada como elementos prioritarios para alcanzar resultados excelentes. A su vez, legitima la priorización de las actividades primordiales respecto de otras de carácter secundario.

Por tanto, Castillo y Nobell (2015) indicarán que la evaluación es una fase clave del sistema de dirección por objetivos orientada a la valoración del cumplimiento, rendir cuentas del desempeño e instrumentar mejoras a partir del trayecto recorrido. De igual forma, evidenciarán que las prácticas de comunicación adolecen de una gran autocomplacencia en sus campañas y en las investigaciones de resultados puesto que todavía no existe una generalización de la necesidad de incrementar y mejorar la valoración y eficacia de las actividades de comunicación.

Medir y evaluar los resultados del trabajo de comunicación es fundamental de acuerdo con la Asociación de Empresas Consultoras en Relaciones Públicas y Comunicación (Adecec, 2016) que indica que lo que no se mide no se puede ni valorar ni gestionar $y$, por consiguiente, no se puede mejorar. Solo midiendo se puede analizar qué errores se han cometido o qué es verdaderamente eficaz para poder seguir trabajando en pro de unos resultados óptimos.

A partir de este contexto, esta investigación tiene como objetivo realizar un análisis del uso e implementación de la fase de evaluación en las prácticas de los profesionales de comunicación y relaciones públicas a partir de los resultados de los Communication Monitor en Europa, Asia - Pacífico y América Latina, principal estudio de reconocimiento mundial que mide las tendencias en comunicación.

El análisis establece un acercamiento a las consideraciones recientes en las prácticas profesionales en evaluación de la comunicación a partir de factores como los niveles de medición y los métodos más usados, la evolución de la fase de la evaluación en las prácticas profesionales y las principales acciones que se derivan a partir de los resultados obtenidos por ésta.

\section{Metodología}

Se utilizará el análisis de contenido como técnica de interpretación de textos e instrumento de recogida de información para realizar una lectura sistemática, objetiva, replicable y válida (Abela, 2000). Se realizará una revisión de las 10 versiones del European Communication Monitor (2007-2017), al igual que del Asia - Pacific Communication Monitor (2015-2016, 2017-2018) y el Latin American Communication Monitor (2016 - 2017) analizando los componentes de la perspectiva de evaluación identificados por los estudios.

Este análisis se establecerá teniendo en cuentas tres variables. La primera los niveles de medición y evaluación predilectos a partir de las categorías de outputs, inputs, outcomes y outflows del modelo del German Public Relations Association / International Controller Association (DPRG/ICV). La segunda, la evolución de la práctica de la evaluación y la tercera, las acciones emprendidas a partir de los resultados de la evaluación. 
Para este propósito se eligió la técnica de análisis de contenido porque combina la observación y producción de los datos, y la interpretación y análisis de los mismos. El análisis de contenido es definido por Berelson (1952, p. 18 citado por Abela, 2000) como una técnica de investigación para la descripción objetiva, sistemática y cuantitativa del contenido manifiesto de la comunicación.

La objetividad se refiere al uso de procedimientos que puedan ser utilizados por otros investigadores de modo que los resultados obtenidos puedan ser verificados. La sistematización indica pautas ordenadas que abarquen el total del contenido observado. La cuantificación pide que se pueda detallar numéricamente la información para obtener el recuento de las unidades o indicadores de los fenómenos de estudio. Y por último, que el contenido sea manifiesto, es para asegurar que la codificación de los datos en el análisis sea verificable y fiable (Abela, 2000).

\section{Resultados}

Una práctica efectiva de comunicación se basa según formula Meng y Pan (2012) en una mayor comprensión del efecto que las iniciativas y estrategias de comunicación pueden tener en la orientación estratégica global de la organización. En el desafiante clima económico actual, los equipos de comunicación se encuentran bajo una creciente presión para mejorar y maximizar los retornos de las comunicaciones sobre el valor de la organización.

Esta demanda se ha visto reflejada en los resultados del European Communication Monitor, ECM, 2016 (Zerfass, Verhoeven, Moreno, Tench \& Verčič, 2016) que definió la vinculación de la estrategia empresarial con la gestión de la comunicación en las organizaciones como el aspecto estratégico más importante a considerar en la gestión de comunicación hasta el año 2019 , lo cual representa una oportunidad de trabajo y de mejora para aquellas organizaciones que aún no han alcanzado un óptimo nivel de alineación.

Esto se ve reflejado concretamente en las prácticas de comunicación y en cómo los profesionales europeos invierten su tiempo productivo en el trabajo. El estudio reporta que tan solo el $18,8 \%$ de los encuestados lo utiliza para la gestión de la comunicación reflexiva que hace referencia a la alineación de la comunicación con la organización y las relaciones con los públicos. Por tanto, poco tiempo queda en la agenda diaria en las organizaciones para identificar objetivos organizacionales, estudiar las expectativas de los públicos, monitorear los temas de asuntos públicos de interés, analizar informes y estrategias de negocios con la alta dirección, así como discutir sobre nuevos escenarios y oportunidades corporativas.

Mientras que la mayor parte del tiempo (36.2\%) se invierte en la comunicación operativa que conlleva hablar con colegas y periodistas, escribir comunicados de prensa, producir impresos, contenidos en línea y material para otros medios, monitorear los resultados de las actividades y organizar eventos, entre otros (Zerfass et al., 2016).

Resultados similares se obtuvieron en el Latin Communication Monitor, LCM, 2016-2017 (Moreno, Molleda, Athaydes, Suárez, Herrera \& Álvarez, 2017) y en el Asia Pacific Communication Monitor, APCM, 2017-2018 (Macnamara, Lwin, Adi, \& Zerfass, 2017). En el caso de los profesionales de América Latina, que incluyen en el estudio a países de Sur América, Centro América 
y Norte América (solo México); apenas el $20.8 \%$ del tiempo se emplea para alinear la comunicación con la organización, los clientes y demás públicos. La mayor parte del tiempo se dedica a la comunicación operacional (34.3\%) y a gestionar las actividades de comunicación y de apoyo a colaboradores $u$ otras dependencias (27.5\%).

Para el caso de los profesionales de los principales países asiáticos y por el pacífico a los de Australia, Fiji y Nueva Zelanda; también tan solo un $20.5 \%$ del tiempo será empleado en labores de alineación de la comunicación con la organización, mientras que un 32.8\% en comunicación operacional. Un comparativo global (Figura 1) elaborado por Macnamara et al., (2017) dará cuenta de las similitudes en el tiempo invertido tanto en la comunicación operativa como en el reducido tiempo para labores de alineación más estratégicas.

\section{Figura 1. Comparación global. Tiempo productivo de los profesionales.}

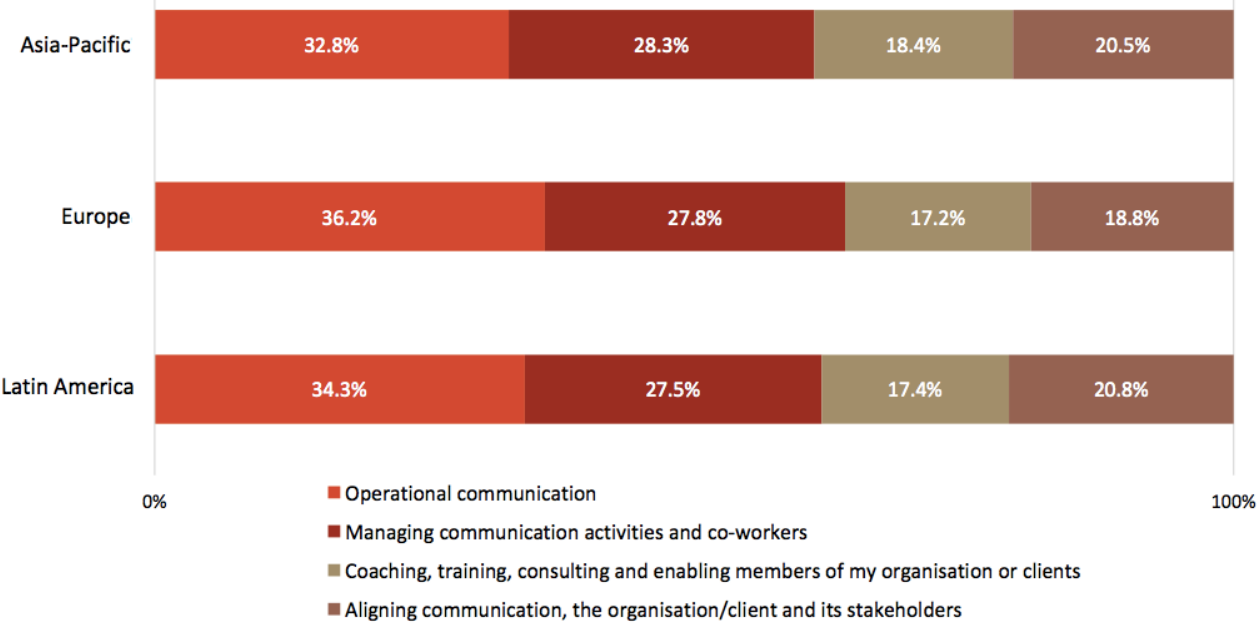

Fuente: Macnamara et al., 2017

Por ende, ante una mayor dedicación de tiempo a labores de generación de contenidos y mantenimiento de medios y canales de comunicación dirigidos hacia los diferentes públicos, propio de la comunicación operacional, habrá una mayor orientación hacia la medición de outputs.

Así se evidencia en el ECM 2015 (Zerfass, Verčič, Verhoeven, Moreno \& Tench, 2015) al indicar que de acuerdo con los cuatro niveles de evaluación en comunicación del modelo DPRG/ICV (Figura 2) el $61.9 \%$ de los profesionales evalúan los outputs, $56.4 \%$ los inputs, $49.7 \%$ los outcomes y tan solo el $37.5 \%$ los outflows. Por tanto, según la clasificación de indicadores empleados en el estudio (Tabla 1), la mayoría llevan a cabo el monitoreo de seguimiento de medios (82.4\%), uso y acceso de internet/intranet (68.9\%), costos financieros del proyecto $(67.8 \%)$ y satisfacción de los clientes internos (57.5\%). 


\section{Figura 2. Niveles de impacto y evaluación de comunicación.}

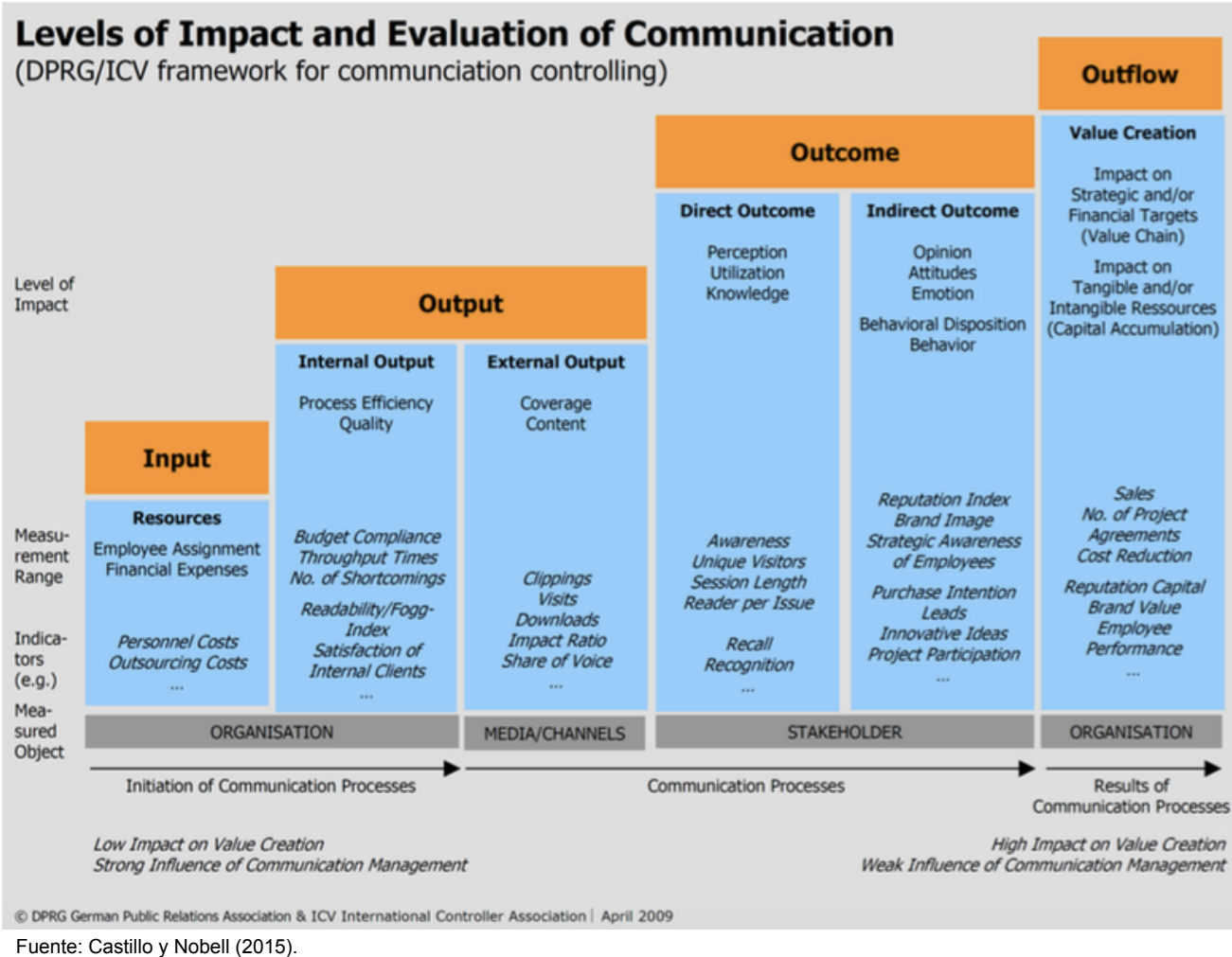

A continuación, se mide el entendimiento de los mensajes claves (53.6\%), las actitudes y cambios de comportamiento de los públicos (45.8\%) y los costos del personal vinculado al mismo (44.9\%). En último lugar, estarán el impacto y los efectos generados en los objetivos financieros y estratégicos de la organización (39.4\%) y en los recursos tangibles e intangibles (35.6\%).

Tabla 1. Clasificación de indicadores de medición Communication Monitor (Modelo DPRG/ICV)

\begin{tabular}{|c|l|l|}
\hline Nivel & \multicolumn{1}{|c|}{ Descripción } & \multicolumn{1}{c|}{ Indicador } \\
\hline $\begin{array}{c}\text { Input } \\
\text { (Organización) }\end{array}$ & $\begin{array}{l}\text { Recursos económicos, físicos y } \\
\text { humanos para iniciar un programa } \\
\text { de relaciones públicas. }\end{array}$ & $\begin{array}{l}\text { - Costos financieros del proyecto } \\
\text { - Costos personal vinculado }\end{array}$ \\
\hline $\begin{array}{c}\text { Output } \\
\text { (Medios y canales) }\end{array}$ & $\begin{array}{l}\text { Resultados inmediatos de una } \\
\text { acción o del programa en términos } \\
\text { de productos, medios y cobertura } \\
\text { del contenido. }\end{array}$ & $\begin{array}{l}\text { - Seguimiento de medios } \\
\text { - Uso de internet/intranet } \\
\text { - Satisfacción de los clientes internos }\end{array}$ \\
\hline
\end{tabular}




\begin{tabular}{|c|l|l|}
\hline $\begin{array}{c}\text { Outcome } \\
\text { (Efectos en los } \\
\text { públicos) }\end{array}$ & $\begin{array}{l}\text { Efectos generados del mensaje } \\
\text { sobre percepción, utilización, } \\
\text { conocimiento, recuerdo e influencia } \\
\text { en la opinión, emociones, actitudes } \\
\text { y comportamiento del público. }\end{array}$ & $\begin{array}{l}\text { - Entendimiento de los mensajes cla- } \\
\text { ves } \\
\text { - Actitudes y cambios de comporta- } \\
\text { miento de los públicos }\end{array}$ \\
\hline $\begin{array}{c}\text { Outflow } \\
\text { (Efectos en la } \\
\text { organización) }\end{array}$ & $\begin{array}{l}\text { Impacto en la creación de valor } \\
\text { y financieros, al igual en los } \\
\text { recursos tangibles e intangibles de } \\
\text { la organización. }\end{array}$ & $\begin{array}{l}\text { - Impacto y efectos generados en obje- } \\
\text { - Impacto en recursos tangibles e in- } \\
\text { tangibles }\end{array}$ \\
\hline
\end{tabular}

Fuente: Elaboración propia a partir de Zerfass, et al., (2015).

El ECM 2015, que en esa versión abordó la temática de evaluación y medición, realiza un análisis longitudinal de la evolución de los métodos de medición usados por los profesionales y registrados en el European Communication Monitor del año 2010 y comparó frente a los del 2015. Los resultados no muestran grandes diferencias con los descritos anteriormente.

Se ha mantenido durante este período una fuerte tendencia a medir y hacer seguimiento principalmente de los medios de comunicación, uso y acceso de internet/intranet y la satisfacción de los clientes internos (outputs). Los mayores crecimientos se registran en la necesidad de medir los inputs utilizados para el desarrollo del proyecto en términos de los costos financieros que pasó de $46.7 \%$ a un $67.8 \%$ y de los costos del personal vinculado que se ubicó inicialmente en un $25.7 \%$ y luego en $44.9 \%$ (Figura 3 ).

Si bien sigue siendo un nivel bajo, se registra también un aumento en la necesidad de medir el impacto que tiene la comunicación en los objetivos financieros y estratégicos de la organización, pasando de un $26.2 \%$ a un $39.4 \%$ y en los recursos tangibles e intangibles, de $24.9 \%$ a $35.6 \%$ (outflows). Zerfass, et al., (2015) expondrán que este último resultado es contradictorio porque a pesar de ser de los factores menos medidos, el $75.5 \%$ de los profesionales encuestados en el estudio considera que la mayor contribución a los objetivos organizacionales es mediante la construcción de activos inmateriales e intangibles precisamente como las marcas, la reputación y la cultura organizacional (outflows). Será difícil comprender cómo los comunicadores hacen este trabajo, si solo una pequeña parte de ellos mide el impacto.

Por otra parte, los profesionales encuestados en el APCM 2015-2016 (Macnamara, Lwin, Adi y Zerfass, 2015) le darán una importancia mayor a la medición de los outflows con un $55.3 \%$ y a los outcomes con $69.9 \%$, con una leve diferencia por encima de los outputs que registran un $66.8 \%$ y en último los inputs con $55.2 \%$. Para este caso, la mayoría de profesionales monitoreará el seguimiento y respuesta de los medios de comunicación (86\%), el entendimiento de los mensajes claves (74\%), uso de internet/intranet (68\%) y la satisfacción de los clientes internos (66\%). 
Figura 3. Análisis longitudinal: métodos usados en 2010 y 2015.

\begin{tabular}{|c|c|c|c|}
\hline Items monitored or measured & 2010 & 2015 & $\Delta$ \\
\hline $\begin{array}{l}\text { Impact on intangible/tangible resources } \\
\text { (i.e. economic brand value) }\end{array}$ & $24.9 \%$ & $35.6 \%$ & $10.7 \%$ \\
\hline $\begin{array}{l}\text { Impact on financial/strategic targets } \\
\text { (i.e. with scorecards, strategy maps) }\end{array}$ & $26.2 \%$ & $39.4 \%$ & $13.2 \%$ \\
\hline Stakeholder attitudes and behaviour change & $40.9 \%$ & $45.8 \%$ & $4.9 \%$ \\
\hline Understanding of key messages & $52.4 \%$ & $53.6 \%$ & $1.2 \%$ \\
\hline Clippings and media response & $82.0 \%$ & $82.4 \%$ & $0.4 \%$ \\
\hline Internet / Intranet usage & $72.2 \%$ & $68.9 \%$ & $-3.3 \%$ \\
\hline Satisfaction of internal clients & $55.5 \%$ & $57.5 \%$ & $2.0 \%$ \\
\hline Process quality (internal workflow) & $26.0 \%$ & $38.6 \%$ & $12.6 \%$ \\
\hline Financial costs for projects & $46.7 \%$ & $67.8 \%$ & $21.1 \%$ \\
\hline Personnel costs for projects & $25.7 \%$ & $44.9 \%$ & $19.2 \%$ \\
\hline
\end{tabular}

Fuente: Zerfass, et al., (2015)

En segundo lugar, medirán las actitudes y cambios de comportamiento de los públicos (65\%), los costos financieros del proyecto (65\%), el impacto y los efectos generados de los recursos tangibles e intangibles (56\%) y en los objetivos financieros y estratégicos de la organización (55\%). Por último, estarán los costos del personal vinculado (46\%).

Al comparar los resultados de este último Communication Monitor de Asia - Pacífico frente al de Europa (Figura 4), denota como punto común en las dos regiones la relevancia principal por medir y hacer seguimiento a los medios de comunicación en cuanto al número de apariciones o menciones que tiene la organización allí (outputs). Y como diferencia, radica la mayor preponderancia que otorga Asia - Pacífico por evaluar los efectos que tienen los mensajes en los públicos en términos de percepción, conocimiento e influencia en la conducta o comportamiento (outcomes). Al igual que del impacto que tienen las acciones de comunicación y relaciones públicas en el logro de los objetivos empresariales (outflows).

Zerfass, et al., (2015) señalarán que si bien la evaluación que se realiza en los cuatro niveles (input, output, outcome y outflow) es importante, demostrar la generación de valor que producen las actividades de comunicación a la organización es más transparente si se hace desde los niveles de outcome y outflow. 


\section{Figura 4. Comparativo niveles de evaluación y medición.}

$$
\text { Europa }=\text { Asia - Pacífico }
$$

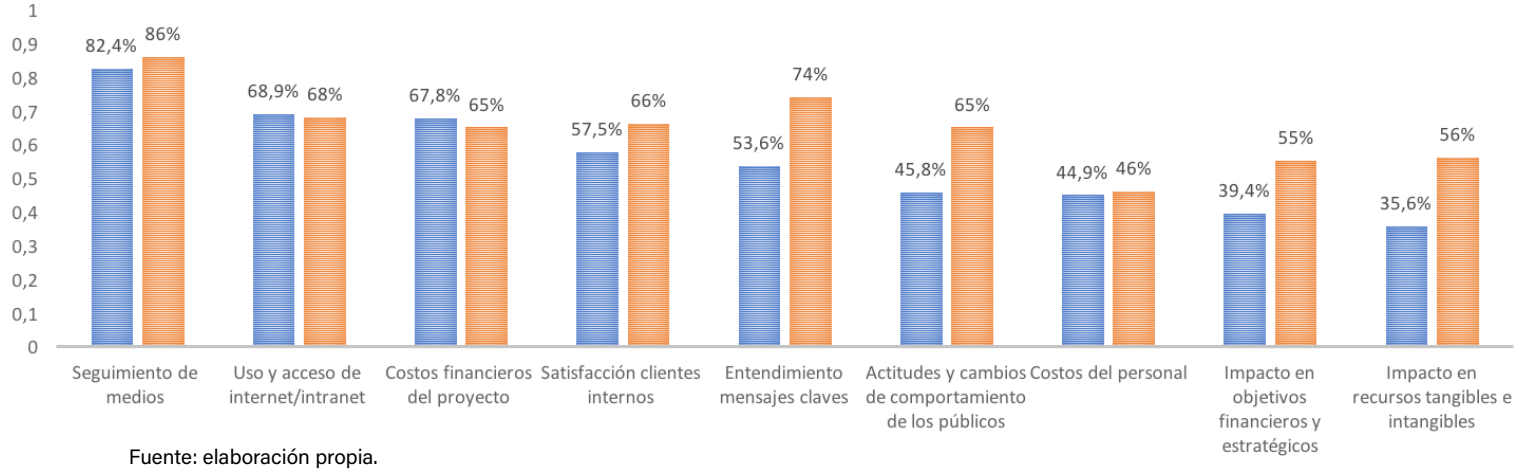

De igual forma, mencionarán que la inconsistencia entre lo que los comunicadores pretenden hacer y cómo explican sus tareas a la alta dirección, por un lado, y los niveles de monitoreo y medición de las mismas actividades, por otro, puede ser una explicación de por qué vincular la estrategia empresarial con el trabajo de comunicación sigue siendo la temática más importante en la gestión de la comunicación en los últimos años. Citando a Watson (2012) señalarán que una de las soluciones a este problema es simple: para poder demostrar la generación de valor empresarial, hay que medir lo que se hace.

La necesidad de conectar las estrategias de la organización y la comunicación es un reto que se ha evidenciado desde la versión 2010 del ECM, en donde ya se indicaba que la falta de medición en el nivel outflow era coherente con la fuerte búsqueda de los profesionales por establecer un vínculo directo con las metas organizacionales (Zerfass, Tench, Verhoeven, Verčič \& Moreno, 2010). Un propósito que seguirá en la agenda del sector, puesto que el $37.5 \%$ de los comunicadores encuestados en la versión 2017 del estudio, lo definieron como el segundo tema más estratégico en la gestión de la comunicación hasta el 2020, tan solo superado con un 40.4\% que considera en primer lugar, hacer frente a la evolución digital y la web social (Zerfass, Moreno, Tench, Verčič, \& Verhoeven, 2017b).

Un resultado similar se presenta en Asia - Pacífico donde vincular las estrategias de la organización y la comunicación también ocupará una segunda posición con un 34.9\% dentro de las prioridades fijadas al 2020 (Macnamara, et al., 2017).

En el caso de América Latina, obtendrá una posición media ocupando el quinto lugar con un 31.7\% en los temas estratégicos al 2019 (Moreno, et al., 2017). Por consiguiente, son Europa y Asia - Pacífico las regiones en donde más protagonismo y prospectiva tiene este aspecto en el debate profesional a corto y mediano plazo como detalla un comparativo global (Figura 5) realizado por Macnamara, et al., (2017). El tema prioritario en las tres regiones será hacer frente a la evolución digital y la web social. 
En una línea similar estará el Global Communications Report, GCR, 2017 (University of Southern California, 2017) que consultó a los profesionales cómo creían que la comunicación podría aumentar su valor dentro de la organización y obtuvo como respuesta mayoritaria con $77 \%$ el demostrar cómo los programas de comunicación alcanzan objetivos de negocio medibles enfocados en variables menos tangibles como la reputación de marca o la intención de compra, entre otros.

A su vez, dentro del listado también se considera la necesidad de mejorar la medición de los resultados de las estrategias y programas, con un $34 \%$.

Figura 5. Comparativo global. Temas estratégicos para la comunicación.

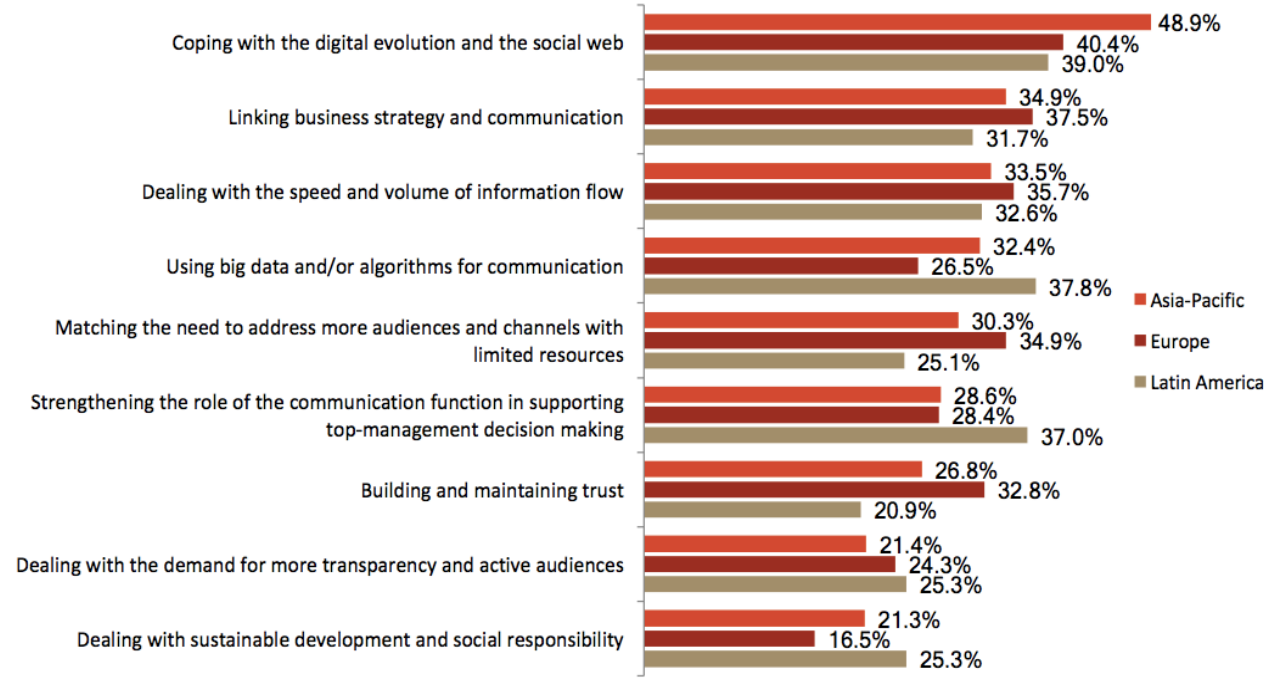

Fuente: Macnamara et al., 2017.

Así mismo, los resultados del ECM 2015 (Zerfass, et al., 2015) encontraron que a la hora de explicar cuál es el valor que tiene la comunicación y su relevancia ante la dirección y los públicos internos, los profesionales argumentaron mayoritariamente que será por medio de la contribución a los efectos positivos de la buena reputación, la cultura organizacional y la marca de la organización (79.8\%). Este argumento también se ubica en la primera posición en Asia - Pacífico donde coincide con el mismo resultado (79.8\%) (Macnamara, et al., 2015).

Adicionalmente, los profesionales en Europa (Zerfass, et al., 2017b) responderán que entre los principales aportes que puede realizar un departamento de comunicación para contribuir al éxito y buena gestión organizacional se encuentran: apoyar los objetivos operacionales y los procesos de otros departamentos a través de actividades de comunicación (86.8\%), al igual que traducir la estrategia organizacional en una estrategia de comunicación totalmente alineada (84.5\%) y comunicar la estrategia organizacional a todos los públicos (82.2\%). 
Una tendencia proporcional se reflejará en los resultados de Asia - Pacífico ubicándose en los tres primeros lugares: apoyar los objetivos operacionales y los procesos de otros departamentos $(80.7 \%)$, traducir la estrategia organizacional en una estrategia de comunicación $(77.7 \%)$ y comunicar la estrategia organizacional a todos los públicos (76.2\%). En este aspecto, todas las variables consideradas como contribuciones de la comunicación en Europa estarán por encima de las de Asia - Pacífico (Figura 6).

Figura 6. Contribuciones de la comunicación.

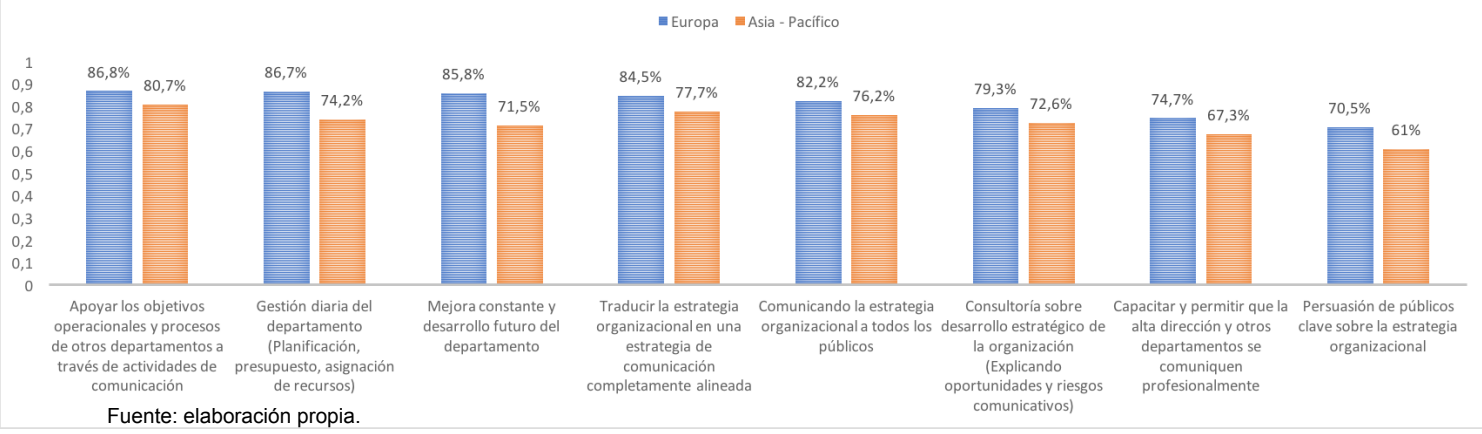

A partir de estos resultados, Zerfass, et al., (2017b) describirán que recientes investigaciones (citando a Volk et al., 2017; Zerfass \& Volk, 2017) han sistematizado estas contribuciones que realizan los departamentos de comunicación en cuatro grupos: transmitir y multiplicar, alinear y contribuir, dirigir y gestionar y asesorar y entrenar; con dos dimensiones, una estratégica y otra operacional (Figura 7). Por consiguiente, fundamentarán el ECM 2017 sobre una noción de comunicación estratégica que se caracteriza por su orientación y capacidad de apoyar las metas organizacionales. A pesar de que pueda parecer una posición obvia en el contexto empresarial, el estudio cita a Brønn (2014) al evidenciar que estudios anteriores indican que los comunicadores rara vez informan sobre cómo su departamento contribuye a los objetivos estratégicos generales.

A pesar de estas consideraciones, el estudio en 2017 encontrará también que en Europa los profesionales creen que los altos directivos no son conscientes de todas las posibles contribuciones que pueden ofrecer los departamentos de comunicación a la organización y están más interesados en la función tradicional que realiza el departamento y parecen dudar del rol de la comunicación en el desarrollo estratégico de la organización (Zerfass, et al., 2017b). 


\section{Figura 7.}

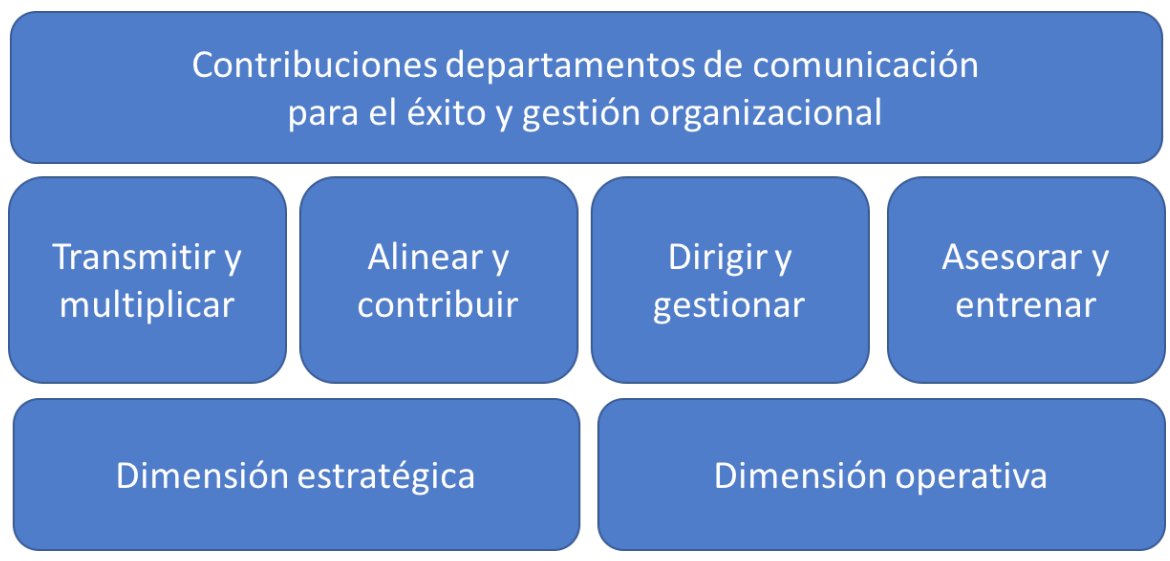

Fuente: Elaboración propia.

En referencia a la evaluación en comunicación los communication monitor de Europa y Asia - Pacífico arrojarán datos adicionales que permitirán contextualizar esta investigación. El primero de ellos está relacionado con la baja posición que ha tenido la evaluación dentro de las temáticas consideradas prioritarias en la gestión de la comunicación para los siguientes años por parte de los profesionales (Figura 8).

En Europa en 2017 tan solo un 16.8\% considera relevante la implementación de rutinas avanzadas de medición y evaluación (Zerfass, et al., 2017b). En la versión 2015 se ubicó en 15.8\% (Zerfass, et al., 2015), en 2010 obtuvo un 17.2\% cuando se incluyó en el listado como establecer nuevos métodos para evaluar la comunicación (Zerfass, et al., 2010), al igual que en 2009 cuando obtiene un 18.8\% (Zerfass, Moreno, Tench, Verčič \& Verhoeven, 2009). En 2008 se ubica en $16.1 \%$ (Zerfass, Moreno, Tench, Verčič \& Verhoeven, 2008) y en 2007 alcanzó un 31.4\%, su registro más alto precisamente en la primera versión del estudio (Zerfass, Van Ruler, Rogojinaru, Verčič \& Hamrefors, 2007).

Algo similar sucede en Asia - Pacífico donde la implementación de rutinas avanzadas de medición y evaluación alcanza un 15.7\% dentro de los retos previstos al 2018 (Macnamara, et al., 2015). Mientras que en el Latin Communication Monitor en sus dos versiones (2014-2015, 20162017) no será mencionado por los profesionales encuestados, evidenciándose una mayor consideración de incluir la evaluación en el debate profesional y académico de los comunicadores en Europa y Asia - Pacífico, a pesar de sus bajos resultados en los estudios. 
Figura 8. Evolución de la evaluación y la medición.

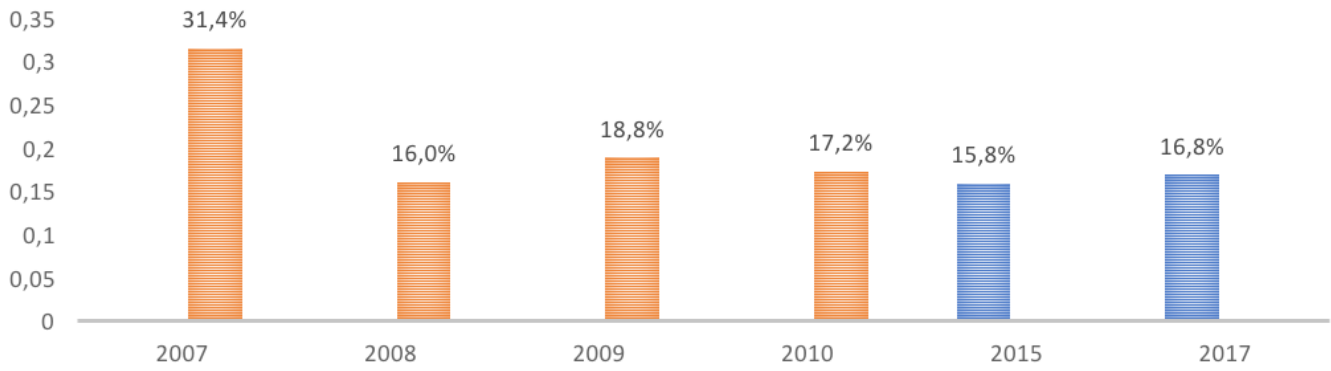

E Implementación de rutinas avanzadas de medición y evaluación Nuevos métodos para evaluar la comunicación

Fuente: Elaboración propia.

Un segundo dato de interés refiere a las acciones y decisiones que los profesionales emprenderán a partir de los resultados generados por la evaluación y medición de sus estrategias y programas de comunicación (Figura 9). En Europa (Zerfass, et al., 2015) la gran mayoría los utilizará para evaluar el éxito de las actividades de comunicación (66\%), hacer la planificación de las próximas actividades (62.9\%), explicar el valor de la comunicación a la dirección y clientes internos (59.5\%), analizar las metas y el direccionamiento de las estrategias de comunicación (58\%) y por último, para liderar equipos de comunicación y el trabajo de agencias externas y proveedores de servicios (43.3\%).

Figura 9. Acciones a partir de los resultados de evaluación y medición.

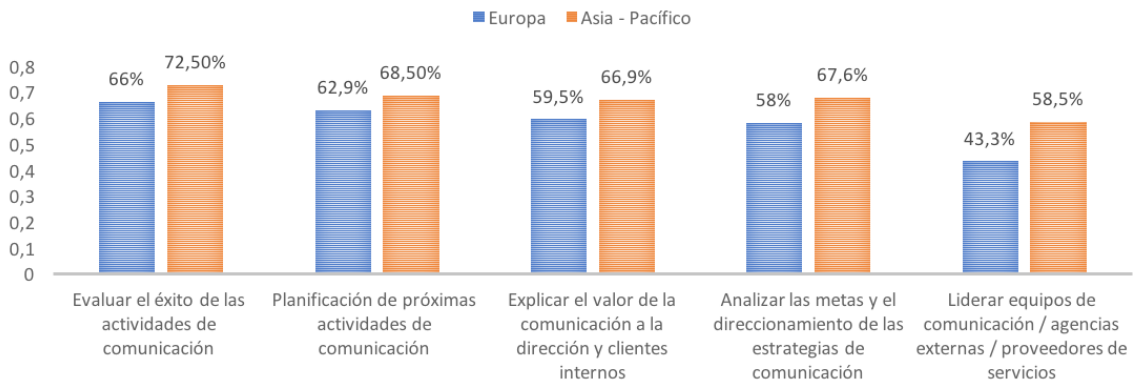

Fuente: Elaboración propia.

En Asia - Pacífico todos los datos estarán por encima de los resultados europeos, dado que tendrá una importancia mayor el evaluar el éxito de las actividades de comunicación (72.5\%), seguido de hacer la planificación de las próximas actividades (68.5\%) en los primeros lugares. Luego se encontrarán analizar las metas y el direccionamiento de las estrategias de comunicación (67.6\%), explicar el valor de la comunicación a la dirección y clientes internos (66.9\%) y liderar equipos de comunicación, agencias externas y proveedores de servicios (58.5\%). 


\section{Discusión}

Los resultados de los Communication Monitor nos permiten establecer las siguientes consideraciones acerca de las prácticas profesionales.

El primero es sobre la relevancia que se le otorga a la fase de evaluación y medición dentro de un programa o campaña de comunicación. Frente a este hecho, los Communication Monitor arrojan un panorama poco alentador dado que evidencia la baja posición que ha tenido la evaluación y medición dentro de las temáticas consideradas prioritarias en la gestión de la comunicación para los siguientes años por parte de los profesionales. En Europa en 2017 tan solo un $16.8 \%$ considera relevante la implementación de rutinas avanzadas de medición y evaluación (Zerfass, et al., 2017b). Una tendencia que se ha mantenido similar en las últimas cinco mediciones en las que ha sido incluida la evaluación.

Igual situación ocurre en Asia - Pacífico donde la implementación de rutinas avanzadas de medición y evaluación alcanza apenas un 15.7\% dentro de los retos previstos al 2018 (Macnamara, et al., 2015).

Otro de los resultados encontrados por el Communication Monitor en Europa refiere a las acciones y decisiones que los profesionales emprenderán a partir de los resultados obtenidos de la evaluación y medición de sus estrategias y programas de comunicación. La gran mayoría los utilizará para evaluar el éxito de las actividades de comunicación (66\%), hacer la planificación de las próximas actividades (62.9\%), explicar el valor de la comunicación a la dirección y clientes internos (59.5\%), analizar las metas y el direccionamiento de las estrategias de comunicación (58\%) y por último, para liderar equipos de comunicación y el trabajo de agencias externas y proveedores de servicios (43.3\%) (Zerfass, et al., 2015). Una situación similar se registra en Asia-Pacífico.

Frente a esto desarrollar un trabajo detallado de análisis y comprensión de insights o de los resultados obtenidos a partir de la medición y evaluación será fundamental dado que permite mejorar los procesos de comunicación, evaluar el cumplimiento y eficacia de los objetivos de los programas y formular mejores campañas de comunicación futuras o ajustar las que estén en marcha. De ahí el interés de las agencias de relaciones públicas y comunicación y de los profesionales de no solo detenerse en la medición sino también en la analítica y en los insights.

En cuanto a evolución digital, la función de medios digitales y sociales es la que cuenta con la mayor demanda de servicios de medición y ha tenido un vertiginoso crecimiento, de este hecho da cuenta el Communication Monitor al ubicar que hacer frente a la evolución digital y la web social será el tema más estratégico en la gestión de la comunicación hasta el año 2020 por parte de los profesionales en Asia-Pacífico (48.9\%), Europa (40.4\%) y América Latina (39\%).

\section{Conclusiones}

Conforme a lo expuesto anteriormente señalaremos que lo descrito antes por Castillo y Nobell (2015) sobre que en la actualidad muy pocas organizaciones evalúan su desempeño en comunicación en relación a los demás procesos de gestión y fundamentalmente, en función de los objetivos organizacionales, siendo una de las funciones menos desarrolladas; se evidencia claramente en los resultados expuestos. 
Por un lado, se encuentra en la baja posición que ha tenido la evaluación dentro de las temáticas consideradas prioritarias en la gestión de la comunicación para los siguientes años por parte de los profesionales encuestados durante los últimos 10 años, teniendo solo un pico alto en el 2007, fecha en la que se realizó el primer estudio. Por otro, se reconoce en la baja medición y evaluación que se realiza de los niveles de outcomes y outflows, concentrándose principalmente los esfuerzos en los outputs o resultados inmediatos que generan los programas de comunicación y relaciones públicas. De lado y en último lugar, quedarán la medición del impacto y los efectos generados en los objetivos financieros y estratégicos de la organización y en sus recursos tangibles e intangibles.

Esto en contradicción precisamente con los factores que precisamente son los que agregan valor a las relaciones públicas y la comunicación que son los intangibles como la reputación, la confianza, la marca, cultura organizacional, identidad, entre otros. Los comunicadores reconocerán siempre que aportar desde su labor a la construcción de éstos será la principal forma de demostrar el valor de su trabajo pero ante ello, la realidad es que el porcentaje de medir y evaluar dicha contribución es muy bajo.

Zerfass, et al., (2015) señalaban que si bien la evaluación y medición se realiza en los cuatro niveles de input, output, outcome y outflow, a la hora de demostrar la generación de valor que producen las actividades de comunicación a la organización es más transparente si se hace desde los niveles de outcome y outflow. Pero después del análisis se evidencia claramente el dominio de la medición al nivel de outputs y como actividad principal el monitoreo y seguimiento de medios.

Otro aspecto a valorar, como indica Zerfass, et al., (2015), es la inconsistencia que se evidencia entre lo que los comunicadores pretenden hacer y en cómo explican sus tareas a la alta dirección, frente a los niveles de monitoreo y medición de las mismas actividades. Lo que será una de las explicaciones del por qué vincular la estrategia empresarial con la gestión de comunicación sigue siendo la temática más importante para la mayoría de comunicadores tanto en Europa como en Asia - Pacífico. Esto corresponde en parte a la gran autocomplacencia que adolecen las campañas e investigaciones de resultados de comunicación y relaciones públicas de la que hablaban Castillo y Nobell (2015) puesto que todavía no existe una generalización de la necesidad de incrementar y mejorar la valoración y eficacia de las actividades de comunicación.

Por otro lado, las investigaciones previas han reflejado que dentro de los principales obstáculos que impiden una mayor práctica de la evaluación y medición, se debe en parte al desconocimiento y falta de mayor preparación por parte de los profesionales en el uso de técnicas y métodos de medición y de investigación social. Esto sumado a la falta también de presupuesto en las compañías y departamentos de comunicación destinado para la investigación y la evaluación, al igual que la falta de tiempo por parte de los profesionales para desarrollar este tipo de labores puesto que la mayoría del tiempo se emplea en tareas rutinarias, operativas y enfocadas en la producción de outputs y en la generación de contenidos para los diferentes canales o medios de comunicación. 


\section{Referencias.}

- Abela, J. A. (2000). Las técnicas de Análisis de Contenido: Una revisión actualizada. Fundación Centro Estudios Andaluces, Universidad de Granada, 10 (2), 1 - 34. Recuperado de: http://public.centrodeestudiosandaluces.es/pdfs/S200103.pdf

- Adecec, Asociación de Empresas Consultoras en Relaciones Públicas y Comunicación. (2016). Guía PRáctica de la Medición. Recuperado de: http://www.adecec.com/noticias/ detalle.php?id=56

- Castillo, A., \& Smolak, E. (2013). Redes sociales y organizaciones. Modelos de evaluación. Historia y Comunicación Social, 18, pp. 473-487. doi: 10.5209/rev-HICS.2013.v18.44343

- $\quad$ Castillo, A., Almansa, A., \& Smolak, E. (2015). East European think tanks in social media - Towards the model of evaluation of effective communication/pr strategies: Case study analysis. Catalan Journal of Communication and Cultural Studies, 7 (2), pp. 231-250. doi: 10.1386/cjcs.7.2.231_1

- $\quad$ Castillo, A., \& Nobell, A. (2015). Evaluación en comunicación estratégica. Madrid: McGraw-Hill.

- Macnamara, J., Lwin, M. O., Adi, A., \& Zerfass, A. (2015). Asia-Pacific Communication Monitor 2015/16. The state of strategic communication and public relations in a region of rapid growth. Survey results from 23 countries. Recuperado de: http://www.zerfass.de/ APCM-WEBSITE/media/APCM-2015-16-Report.pdf

- Macnamara, J., Lwin, M.O., Adi, A., \& Zerfass, A. (2017). Asia-Pacific Communication Monitor 2017/18. Strategic challenges, social media and professional capabilities. Results of a survey in 22 countries. Recuperado de: http://www.zerfass.de/APCM-WEBSITE/media/ APCM-2017-18-Report.pdf

- Marca, G., Oliveira A., Matilla K. \& Miranda T. (2017). El valor de la evaluación de las relaciones públicas y de la comunicación en las organizaciones: el caso de los hospitales del Sistema Nacional de Salud Español. Palabra Clave, 20(2), 506-528. doi: 10.5294/pacla.2017.20.2.9

- Meng, J., \& Pan, P. (2012). Using a balanced set of measures to focus on long-term competency in internal communication. Public Relations Review, 38 (3), pp. 484-490. doi: 10.1016/j.pubrev.2012.03.005

- Moreno, A., Molleda, J., Athaydes, A., Suárez, A., Herrera, M. \& Álvarez, A. (2017). Latin American Communication Monitor 2016 - 2017. Tendencias en comunicación estratégica: big data, automatización, engagement, influencers, coaching y competencias. Resultados de una encuesta en 17 países. Recuperado de: http://latincommunicationmonitor.com/ site/2016-2017/

- Zerfass, A., Van Ruler, B., Rogojinaru, A., Verčič, D., \& Hamrefors, S. (2007). European Communication Monitor 2007. Trends in Communication Management and Public Relations Results and Implications. Recuperado de: http://www.zerfass.de/ECM-WEBSITE/media/ ECM2007-Results.pdf

- Zerfass, A., Moreno, A., Tench, R., Verčič, D., \& Verhoeven, P. (2008). European Communication Monitor 2008. Trends in Communication Management and Public Relations - Results and Implications. Recuperado de: http://www.zerfass.de/ECM-WEBSITE/media/ ECM2008-Results.pdf

- Zerfass, A., Moreno, A., Tench, R., Verčič, D., \& Verhoeven, P. (2009). European Communication Monitor 2009. Trends in Communication Management and Public Relations - Results of a Survey in 34 Countries. Recuperado de: http://www.zerfass.de/ECM-WEBSITE/ media/ECM2009-Results-ChartVersion.pdf 
- Zerfass, A., Tench, R., Verhoeven, P., Verčič, D., \& Moreno, A. (2010). European Communication Monitor 2010. Status Quo and Challenges for Public Relations in Europe. Results of an Empirical Survey in 46 Countries. Recuperado de: http://www.zerfass.de/ECM-WEBSITE/media/ECM2010-Results-ChartVersion.pdf

- Zerfass, A., Verčič, D., Verhoeven, P., Moreno, A., \& Tench, R. (2015). European Communication Monitor 2015. Creating communication value through listening, messaging and measurement. Results of a Survey in 41 Countries. Recuperado de: http://www.zerfass. de/ECM-WEBSITE/media/ECM2015-Results-ChartVersion.pdf

- Zerfass, A., Verhoeven, P., Moreno, A., Tench, R., \& Verčič, D. (2016). European Communication Monitor 2016. Exploring trends in big data, stakeholder engagement and strategic communication. Recuperado de: http://www.zerfass.de/ECM-WEBSITE/media/ ECM2016-Results-ChartVersion.pdf

- Zerfass, A., Verčič, D. \& Volk, S. (2017a). Communication evaluation and measurement: Skills, practices and utilization in European organizations. Corporate Communications: An International Journal, Vol. 22 Issue: 1, pp. 2-18. doi.org/10.1108/ CCIJ-08-2016-0056

- Zerfass, A., Moreno, Á., Tench, R., Verčič, D., \& Verhoeven, P. (2017b). European Communication Monitor 2017. How strategic communication deals with the challenges of visualisation, social bots and hypermodernity. Results of a survey in 50 Countries. Recuperado de: http://www.zerfass.de/ECM-WEBSITE/media/ECM2017-Results-ChartVersion.pdf

\section{CURRICULUM VITAE}

\section{Antonio Castillo Esparcia}

Catedrático de Universidad. Docente en la Universidad de Málaga. Especialista Relaciones Públicas, Comunicación Política e Investigación en Comunicación. Autor de más de 150 publicaciones entre artículos en revistas, libros y capítulos de libros en editoriales nacionales y extranjeras. Director del Proyecto de Investigación Lobby y Comunicación, financiado por el Programa Estatal de I+D+i (CSO2016-79357-R). Director del Grupo de Investigación Las Relaciones Públicas en la pequeña y Mediana Empresa.

\section{Diego Villabona Beltrán}

Comunicador Social Organizacional, distinción cum laude y Premio Extraordinario Fin de Máster en Dirección Estratégica e Innovación en Comunicación de la Universidad de Málaga. Director de Comunicaciones y Relaciones Públicas de Crezcamos microfinanciera en Colombia. Con experiencia en diseño y ejecución de estrategias de comunicación y relacionamiento en función de los diferentes públicos y objetivos corporativos. 